\title{
A New Speech Enhancement Technique to Reduce Residual Noise Using Perceptual Constrained Spectral Weighted Factors
}

\author{
K.Ravi Kumar, T.Munikumar \\ Faculty Member, Dept.of ECE,Gudlavalleru Engineering College, Gudlavalleru-521356, AP, India \\ Faculty Member, Dept.of ECE, Amrita sai Engineering College, Paritala-521356, AP, India.
}

\begin{abstract}
This paper deals with residual musical noise which results from the perceptual speech enhancement type algorithms and especially using wiener filtering approach. Perceptual speech enhancement techniques perform better than the non perceptual techniques, most of them still return a trouble residual musical noise. This is due to that only noise above the noise masking threshold (NMT) is filtered out then noise below the noise masking threshold (NMT) can become audible if its maskers are filtered. It can affect the performance of perceptual speech enhancement method that process the audible noise only (Residual noise is still present). In order to overcome this drawback a new speech enhancement technique is proposed here. The main aim here is to improve the enhanced speech signal quality provided by perceptual wiener filtering and by controlling the latter via a second filter regarded as a psychoacoustically motivated weighting factor. The simulation results gives the information that the performance is improved compared to other perceptual speech enhancement methods.
\end{abstract}

\section{Introduction}

The objective of speech enhancement is to improve the intelligibility and quality of speech in noisy environments. Many approaches have been proposed like spectral subtractive type [1-4], Perceptual Wiener filtering types. Among them spectral subtraction and the Wiener filtering algorithms are using frequently because of their low computational complexity and outstanding performance. In these algorithms, Such methods leaves residual noise known as musical noise. This type of noise is quite annoying(trouble). In order to reduce the effect of musical noise, several methods and solutions have been proposed. Some of them involve adjusting the parameters of spectral subtraction so as to give more flexibility as in [2] and [3]. Other techniques such as proposed in [4], are based on signal subspace approaches. Even though these methods effectively improve the signal to noise ratio (SNR), the problem of eliminating musical noise is still a challenge to many researchers. In the last few decades the introduction of modeling the signal with consideration of psychoacoustic has attracted a great deal of interest. The main objective is to improve the perceptual quality of the enhanced signal. In [3], a psychoacoustic model is used to change or control the parameters of the spectral subtraction in order to find the best trade off between speech distortion and noise reduction. To make musical noise inaudible, the proposed technique linear estimator in [5] uses the human auditory system and its masking properties. In [6], the intermediate signal and masking threshold, which is slightly denoised and free of musical noise, are used to detect musical tones generated by the spectral subtraction methods. This detection can be used by a postprocessing techniques which aims at reducing the detected tones. These perceptual speech enhancement systems reduce the residual noise but introduce some undesired distortion to the enhanced speech signal. When this distorted estimated speech signal(enhanced) is applied to the recognition systems their performance degrades drastically.

The idea of the proposed method is to remove, perceptually significant noise components from the noisy or corrupted signal, so that the clean speech components are not affected by processing (any enhancing process). In addition, the technique requires very little a priori information (information before processing the noisy) signal of the features of the noise. In the present paper, we propose to control the perceptual wiener filtering by psychoacoustically motivated filter that can be regarded as weighting factor. The purpose is to minimize or reduce the perception of residual noise without degrading the clarity of the enhanced speech signal.

\section{II. standard speech enhancement technique}

Let the noisy signal can be expressed as

$$
y(n)=x(n)+d(n),
$$

Where $x(n)$ is the original clean speech signal and $d(n)$ is the additive random noise signal, uncorrelated with the original signal. Taking DFT to the observed signal gives

$$
Y(m, k)=X(m, k)+D(m, k) \text {. }
$$


Where $m=1,2, \ldots, M$ is the frame index, $k=1,2, \ldots, K$ is the frequency bin index, $M$ is the total number of frames and $K$ is the frame length, $Y(m, k), X(m, k)$ and $D(m, k)$ represent the short time spectral components of the $y(n), x(n) \operatorname{and}(n)$, respectively. Clean speech spectrum $\hat{X}(m, k)$ is obtained by multiplying noisy speech spectrum with filter gain function as given in eqation (3)

$$
\hat{X}(m, k)=H(m, k) Y(m, k)
$$

Where $H(m, k)$ is the noise suppression filter gain function Wiener filter (WF)), which is derived according to MMSE estimator and $H(m, k)$ is given by

$$
H(m, k)=\frac{\xi(m, k)}{1+\xi(m, k)}
$$

Where $\xi(m, k)$ is an apriori SNR, which is defined as

$\xi(m, k)=\frac{\Gamma_{x}(m, k)}{\Gamma_{d}(m, k)}$. (5) $\quad \Gamma_{d}(m, k)=E\left\{\left.D(m, k)\right|^{2}\right\}$ and $\Gamma_{x}(m, k)=E\left\{\left.x(m, k)\right|^{2}\right\}$ represents the estimated noise power spectrum and clean speech power spectrum. A posteriori estimation is given by

$$
\gamma(m, k)=\frac{|Y(m, k)|^{2}}{\Gamma_{d}(m, k)}
$$

An estimate of $\hat{\xi}(m, k)$ of $\xi(m, k)$ is given by the well known decision directed approach [9] and is expressed as

$$
\hat{\xi}(m, k)=\alpha \frac{|H(m-1, k) Y(m-1, k)|^{2}}{\Gamma_{d}}+(1-\alpha) P^{\prime}[V(m, k] .
$$

Where $V(m, k)=\gamma(m, k)-1, \quad P[x]=x$ if $x \geq 0$ and $P[x]=0$ otherwise. The noise suppression gain function is chosen as the Wiener filter same as in[13]

\section{Perceptual Speech Enhancement}

Although the Wiener filtering reduces the level of musical noise, it does not eliminate it [15]. Musical noise exists and perceptually troubles. So as an effort to make the residual noise perceptually inaudible, many perceptual speech enhancement methods have been proposed which incorporates the auditory masking properties [2-9]. In these methods residual noise is shaped according to an estimate of the signal masking threshold $[9,13]$. Figure 1 depicts the complete block diagram of the proposed speech enhancement method.

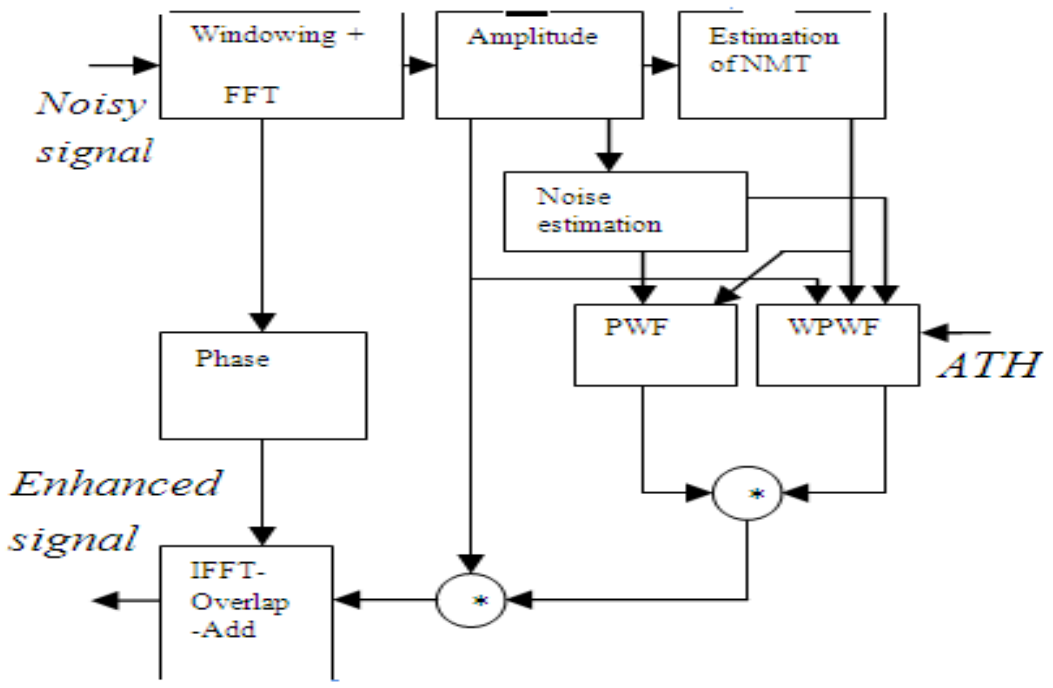

Figure1. Block diagram of the proposed speech enhancement method 


\subsection{Gain of Perceptual Wiener filter (PWF)}

The perceptual Wiener filter (PWF) gain function $H_{1}(m, k)$ is calculated based on cost function, $J$ which is defined as

$$
J=\left[|\hat{X}(m, k)-X(m, k)|^{2}\right]
$$

Substituting (2) and (3) in (9) results to

$$
\begin{aligned}
& =E\left\{\left(H_{1}(m, k)-1\right) X(m, k)+\left.H_{1}(m, k) D(m, k)\right|^{2}\right\} \\
& =d_{i}+r_{i}
\end{aligned}
$$

Where

$d_{i}=\left(H_{1}(m, k)-1\right)^{2} E\left[\left.X(m, k)\right|^{2}\right]$ and $\left.r_{i}=H_{1}^{2}(m, k) E|D(m, k)|^{2}\right]$ represents speech distortion energy and residual noise energy.

To make this residual noise inaudible, the residual noise should be less than the auditory masking threshold, $T(m, k)$. This constraint is given by

$$
r_{i} \leq T(m, k)
$$

By including the above constraint and substituting $\Gamma_{d}(m, k)=E\left\{\left.D(m, k)\right|^{2}\right\}$ and $\Gamma_{x}(m, k)=E\left\{\left.X(m, k)\right|^{2}\right\}$ in (9) the cost function will become as

$J=\left(H_{1}(m, k)-1\right)^{2} \Gamma_{s}(m, k)+H_{1}^{2}(m, k)\left\{\max \left[\left(\Gamma_{d}(m, k)-T(m, k)\right), 0\right]\right\}$

The desired perceptual modification of Wiener is obtained by differentiating $J$ w.r.t $H_{1}(m, k)$ and equating to zero. The obtained perceptually defined Wiener filter gain function is given by $H_{1}(m, k)=\frac{\Gamma_{x}(m, k)}{\Gamma_{x}(m, k)+\max \left(\Gamma_{d}(m, k)-T(m, k), 0\right)}$

By multiplying and dividing equation (12) with $\Gamma_{d}(m, k), H_{1}(m, k)$ will become as

$$
H_{1}(m, k)=\frac{\hat{\xi}(m, k)}{\hat{\xi}(m, k)+\frac{\max \left(\Gamma_{d}(m, k)-T(m, k), 0\right)}{\Gamma_{d}(m, k)}}
$$

$T(m, k)$ is noise masking threshold which is estimated based on[16] noisy speech spectrum. A priori SNR and noise power spectrum were estimated using the two -step a priori SNR estimator proposed in [15] and weighted noise estimation method proposed in[17],respectively.

\subsection{WEIGHTED PWF}

Although perceptual speech enhancement methods perform better than the non-perceptual methods, most of them still return annoying residual musical noise. Enhanced speech signal obtained using above mentioned perceptual Wiener filter still contains some residual noise due to the fact that only noise above the noise masking threshold is filtered and noise below the noise masking threshold is remain. It can affect the performance of perceptual speech enhancement method that processes audible noise only.

In order to overcome this drawback we propose to weight the perceptual Wiener filters using a psychoacoustically motivated weighting filter. Psychoacoustically motivated weighting filter is given by

$W(m, k)=\left\{\begin{array}{l}H(m, k), i f A T H(m, k)<\Gamma_{d} \leq T(m, k) \\ 1, \text { otherwise }\end{array}\right.$

Where $\operatorname{ATH}(m, k)$ is the absolute threshold of hearing. This weighting factor is used to weight the perceptual wiener filter. The gain function of the $H_{2}(m, k)$ of the proposed weighted perceptual Wiener filter is given by

$$
H_{2}=H_{1}(m, k) W(m, k)
$$




\section{Simulation Results}

To evaluate the performance of the proposed scheme of speech enhancement and for comparision, simulations are carried out with the NOIZEUS, A noisy speech corpus for evaluation of speech enhancement algorithms, database [18]. The noisy database contains 30 IEEE sentences (produced by three female and three male speakers) corrupted by eight different real world noises at different SNRs levels. Speech signals were degraded with different types of noise at global SNR levels of $0 \mathrm{~dB}, 5 \mathrm{~dB}, 10 \mathrm{~dB}$ and $15 \mathrm{~dB}$. In this evaluation only five noises are considered those are car, babble, airport, train, and street noise. The objective quality measures used for the evaluation of the proposed speech enhancement method are the PESQ measures and segmental SNR [19]. It is well known that the segmental SNR is more accurate in indicating the speech distortion than the overall SNR. The higher value of the segmental SNR indicates the weaker speech distortion ie less distortion. The higher PESQ score indicates better perceived quality of the proposed signal [19]. The performance of the proposed method is compared with Wiener filter and perceptual Wiener filter.

The simulation results are summarized in Table 1 and Table 2. The proposed method leads to better improvements are obtained for the high noise level and the better denoising quality for temporal. The timefrequency distribution of speech signals provides more accurate information about the residual musical noise and speech distortion than the corresponding time domain waveforms. Here we compared the spectrograms for each of the techniques and confirmed a reduction of the speech distortion and residual noise. Figure2. Represents the spectrograms of the noisy signal, clean speech signal, and enhanced speech signals.

Table.1 Segmental SNR values of Enhanced Signals

\begin{tabular}{|c|c|c|c|c|}
\hline Noise Type & $\begin{array}{l}\text { Input } \\
\text { SNR } \\
\text { (dB) }\end{array}$ & WF & PWF & $\begin{array}{l}\text { Weighted } \\
\text { PWF }\end{array}$ \\
\hline \multirow{4}{*}{ Babble } & 0 & -4.59 & -0.61 & -0.32 \\
\hline & 5 & -1.39 & 0.01 & -0.22 \\
\hline & 10 & 0.02 & 0.65 & 2.14 \\
\hline & 15 & 0.75 & 2.71 & 3.97 \\
\hline \multirow{4}{*}{ Car } & 0 & -3.93 & -0.24 & -0.85 \\
\hline & 5 & -1.65 & 0.52 & 1.20 \\
\hline & 10 & 0.69 & 0.70 & 2.37 \\
\hline & 15 & 0.72 & 2.31 & 3.81 \\
\hline \multirow{4}{*}{ Train } & 0 & -3.45 & -0.49 & -1.15 \\
\hline & 5 & -0.86 & 0.38 & 0.43 \\
\hline & 10 & -0.39 & 0.77 & 2.20 \\
\hline & 15 & 0.75 & 2.62 & 3.5 \\
\hline \multirow{4}{*}{ Airport } & 0 & -4.37 & -0.24 & -0.89 \\
\hline & 5 & -2.57 & 0.15 & 0.43 \\
\hline & 10 & -0.06 & 0.14 & 1.09 \\
\hline & 15 & 0.75 & 1.88 & 3.65 \\
\hline \multirow{4}{*}{ Street } & 0 & -2.88 & -0.15 & -0.08 \\
\hline & 5 & -2.13 & 0.61 & -0.13 \\
\hline & 10 & 0.69 & 1.20 & 2.70 \\
\hline & 15 & 0.77 & 2.25 & 3.42 \\
\hline
\end{tabular}

Table.2 PESQ values of the enhanced signals

\begin{tabular}{|l|c|c|c|c|}
\hline Noise Type & $\begin{array}{l}\text { Input } \\
\text { SNR } \\
(\mathrm{dB})\end{array}$ & WF & PWF & Weighted PWF \\
\hline \multirow{4}{*}{ Babble } & 0 & 1.221 & 0.952 & 1.427 \\
\cline { 2 - 5 } & 5 & 1.728 & 1.750 & 1.836 \\
\cline { 2 - 5 } & 10 & 2.034 & 2.276 & 2.402 \\
\cline { 2 - 5 } & 15 & 2.127 & 2.609 & 2.718 \\
\hline \multirow{4}{*}{ Car } & 0 & 1.165 & 1.439 & 1.734 \\
\cline { 2 - 5 } & 5 & 1.694 & 1.697 & 2.107 \\
\cline { 2 - 5 } & 10 & 1.921 & 2.168 & 2.318 \\
\cline { 2 - 5 } & 15 & 2.265 & 2.645 & 3.127 \\
\hline \multirow{4}{*}{ Airport } & 0 & 1.450 & 1.482 & 1.731 \\
\cline { 2 - 5 } & 5 & 1.680 & 1.715 & 2.133 \\
\cline { 2 - 5 } & 10 & 2.009 & 2.096 & 2.479 \\
\cline { 2 - 5 } & 15 & 2.040 & 2.032 & 2.714 \\
\cline { 2 - 5 } & 0 & 1.472 & 1.561 & 1.759 \\
\cline { 2 - 5 } & 5 & 1.492 & 1.769 & 2.242 \\
\cline { 2 - 5 } & 10 & 2.025 & 2.413 & 2.538 \\
\hline Street & 15 & 2.249 & 2.579 & 2.715 \\
\cline { 2 - 5 } & 0 & 1.636 & 1.782 & 1.817 \\
\cline { 2 - 5 } & 10 & 1.679 & 1.857 & 1.968 \\
\cline { 2 - 5 } & 15 & 2.119 & 2.260 & 2.392 \\
\hline
\end{tabular}




\section{Conclusion}

In this paper, an effective approach for suppressing the musical noise presented after wiener filtering has been introduced. Based on the perceptual properties of the human auditory system, a weighting factor accentuates the denoising process when noise is perceptually insignificant and prevents that residual noise components might become audible in the absence of adjacent maskers. When the speech signal is additively corrupted by babble noise and car noise objective measure results showed the improvement brought by the proposed method in comparison to some recent filtering techniques of the same type.
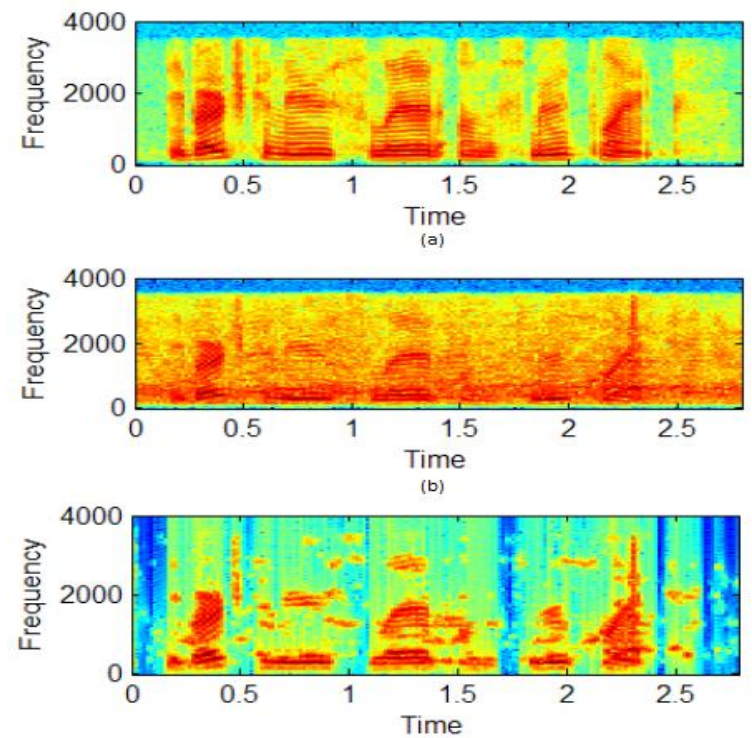

(c)

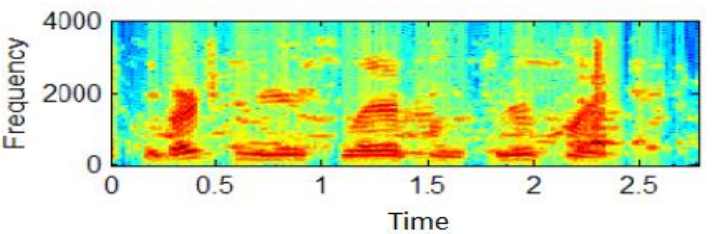

(d)

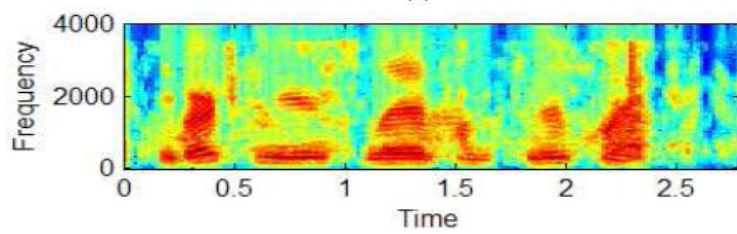

(e)

Figure2. speech spectrogram,(a)original clean signal,(b) noisy signal(babble noise $\mathrm{SNR}=5 \mathrm{~dB}$ ),(c)enhanced signal using Wiener filter(d)enhanced signal using PWF, (e)enhanced signal using Weighted PWF

\section{References}

[1] Y. Ephraim and D. Malah, "Speech enhancement using a minimum mean square error short-time spectral amplitude estimator," IEEE Trans. Acoust., Speech, Signal Processing, vol. ASSP-32, pp. 1109-1121, Dec 1984.

[2] R. Schwartz M. Berouti and J. Makhoul, "Enhancement of speech corrupted by acoustic noise,” Proc. of ICASSP, 1979, vol. I, pp. $208-211$.

[3] N.Virag, "Single channel speech enhancement based on masking properties of the human auditory system," IEEE Trans. Speech and Audio Processing, vol. 7, pp. 126-137, 1999.

[4] Y. Ephraim and H.L. Van Trees, “A signal subspace approach for speech enhancement," IEEE Trans. Speech and Audio Processing, vol. 3, pp. 251-266, 1995.

[5] Y. Hu and P. Loizou, "Incorporating a psychoacoustic model in frequency domain speech enhancement," IEEE Signal Processing Letters, vol. 11(2), pp. 270-273, 2004.

[6] F. Jabloun and B. Champagne, "Incorporating the human hearing properties in the signal subspace approach for speech enhancement," IEEE Trans. Speech and Audio Processing,vol. 11, pp. 700-708, 2003.

[7] Y.M. Cheng and D. O'Shaughnessy, "Speech enhancement based conceptually on auditory Processing, vol.39, no.9, pp.1943-1954, 1991.

[8] D. Tsoukalas, M. Paraskevas, and J. Mourjopoulos, "Speech enhancement using psychoacoustic criteria," IEEE ICASSP, pp.359362, Minneapolis, MN, 1993. 
[9] Y. Hu and P.C. Loizou, "A perceptually motivated approach for speech enhancement," IEEE Trans. Speech Audio Processing, pp. 457-465. Sept. 2003.

[10] L. Lin, W. H. Holmes and E. Ambikairajah, "Speech denoising using perceptual modification of Wiener filtering," IEE Electronic Letters, vol. 38, pp. 1486-1487, Nov 2002.

[11] P. Scalart C. Beaugeant, V. Turbin and A. Gilloire, "New optimal filtering approaches for hands-free telecommunication terminals, "Signal Processing, vol. 64 (15), pp. 33-47, Jan 1998.

[12] T. Lee and Kaisheng Yao, "Speech enhancement by perceptual filter with sequential noise parameter estimation," Proc. of ICASSP, vol. I, pp. 693-696, 2004.

[13] Md. Jahangir Alam, Sid-Ahmed Selouani, Douglas O'Shaughnessy and S. Ben Jebara, "Speech enhancement using a Wiener denoising technique and musical noise reduction" in the Proceeding of INTERSPEECH'08, Brisbane, Australia, pp. 407-410, September 2008 .

[14] Amehraye, D. Pastor, and A. Tamtaoui, "Perceptual improvement of Wiener filtering." Proc. of ICASSP, pp. 2081-2084, 2008.

[15] Md. Jahangir Alam, Douglas O’Shaughnessy and Sid-Ahmed Selouani, "Speech enhancement based on novel two-step a priori SN estimators," in the Proceeding of INTERSPEECH'08, Brisbane,Australia, pp. 565-568, September 2008.

[16] J. D. Johnston, "Transform coding of audio signals using perceptual noise criteria," IEEE on Selected Areas in Comm., vol. 6, pp. 314-323, February1988.

[17] M. Kato, A. Sugiyama and M. Serizawa, "Noise suppression with high speech quality based on weighted noise estimation and MMSESTSA,” IEICE Trans. Fundamentals, vol. E85-A, no.7, pp. 1710-1718, July 2002.

[18] http://www.utdallas.edu/ loizou/speech/noizeus/

[19] Yi Hu and Philipos C. Loizou, "Evaluation of Objective Quality Measures for Speech Enhancement," IEEE Trans. on Audio, Speech and Language Processing, vol. 16, no. 1, pp. 229- 238, January 2008. 